the dose to ten, twenty, and in a few cases to thirty grains, and relied upon its internal administration without the assistance of injections; out of forty cases so treated, an improvement was noticed in twenty within a week, while six were completely cured. Salol is not a mechanical mixture of carbolic and salicylic acids, but appears to be a compound of the nature of an ethereal salt, since phenol can, with some some slight difficulty, be obtained from it on saponification. The potassium salt left after the treatment of salol with caustic potash gives with hydrochloric acid an acid which is either salicylic or one of its isomers. Four hours after administration by the mouth of twenty grains of salol traces of carbolic and distinct evidence of salicylic acids, combined with other elements, were found in the urine. In one case the urine was blackened as in carboluria, the patient at that time taking thirty grains three times a day. It is by the action of the pancreatic juice that salol is said to be split up into carbolic and salicylic acids; of this change I was unable to procure any sufficient evidence after having added pancreatine to a mixture of salol. In salol we have, in my opinion, an addition of considerable value to our list of remedies for gonorrhoea; its effect is apparently produced by the action of the urine upon the inflamed urethra, since that fluid contains salicylate and carbolic acids in combination, probably as salicylurates and sulphocarbolates. It may be given in doses of from ten to twenty grains three times daily at any stage of the disease, and in chronic cases an astringent injection will materially hasten the cure. The effect of the drug does not appear to be enhanced by larger doses than twenty grains; when the amount was increased to thirty grains, the urine became blackened and the symptoms did not show any proportionate improvement. I commenced by giving five-grain doses, but this was soon discovered to be too small a quantity, and my results would, I doubt not, have been more favourable had I commenced its administration with a freer hand. I am indebted to Mr. R. J. Carter, house surgeon to the Male Lock Hospital, for his careful investigation into the chemical properties of the drug, and for nates of the progress of the cases so treated.

Cambridge-street, W.

A CASE OF PYO-NEPHROSIS, COMPLICATED BY MYOSITIS ; DISAPPEARANCE OF THE TUMOUR AFTER AN EXPLORATORY INCISION, WHICH FAILED TO REACH THE KIDNEY.

By Herbert Snow, M.D. Lond., \&c., SURGEON to THE CaNCER hospital.

THE following case affords an example of what would seem to be a very unusual condition; no allusion to which (as a possible complication of nephrotomy) has been found in the numerous papers upon renal operative surgery which have recently appeared. The case is also interesting in view of the remarkable benefit resulting from an incision through the tissues in the neighbourhood (only) of the disease.

Sarah $R$ - aged fifty-six, was admitted into the Cance Hospital on Sept. 30th, 1889, with a rounded tumour of six months' duration to the left of the middle line between the umbilicus and the cartilage of the serenth rib on that side. No fanily history of cancer; no injury; much mental distress and hard work; an emaciated anæmic woman, whose appearance strongly suggested malignant disease. Beside progressive debility she had frequent attacks of acute pain radiating from the tumour; stated that she had frequently passed gravel, and the urine was found to contain large quantities of pus. No special frequency of micturition urine, sp. gr. 10I4; no sugar. The tumour, evidently deepseated, was of about the size of a large orange, at first hard An ill-defined induration was also apparent in the left Iumbar region, filling up the space between the iliac crest and the false ribs. Upon Oct. 13th the patient had a rigor, and the temperature rose to $105^{\circ}$, soon subsiding, how ever, to the normal. Fluctuation in the tumour became apparent, and after a consultation of the staff it was decided to explore the kidney. On Oct. 22nd a three-inch transverse incision was accordingly made about midway between the crest of the ilium and the last rib, although the induration above described made it difficult to trace the bony outlines. The tissues were divided to a depth of from two to three inches. It then became apparent that the museles were, from the iliac crest upwards, in a consolidated gristly condition. The sensation was exactly analogous to that left upon cutting into a scirrhous mammary growth; pro. fuse hæmorrhage also took place, and the original view of malignancy which had been entertained received apparent confirmation. As, moreover, it became obvious that, even if the kidney were actually incised, the rigid brawny state of the parts would preclude the removal of any calculus which might there exist, it was decided not to proceed further. The wound had to be plugged with iron-lint before the bleeding could be arrested. The opera. tion wound quickly healed, and concurrently both the induration and the tumour completely disappeared. By the middle of December complete cicatrisation had taken place deep pressure still caused pain where the mass had been, but the only other trace of the latter was affurded by slight fulness and resistance. Theie was a very marked improvement in the general health, and the woman left at her own request without any bad symptom, being, as she considered, completely restored to health. Some mucus with a very few pus-cells was present (on Jan. 15th last) in the urine. The greater part of the time a mixture of nitro-hydrochloric acid with cinchona was given. Such treatment could obviously have had little share in the favourable result. Gloucester-place, W.

\section{ACUTE INVAGINATION OF COLON.}

BY JohN McMunN, L R C.P. Ed., L.R.C.S.I., LATE DEMONSTRATOR OF ANATOMY, DUBLIN.

A Healthy child was given a dose of castor oil by its mother at 10 A.M. on a Friday morning in September, 1889. The drug was given as a purgative after vaccination; it was not required. Result : three curdy evacuations, slightly slimy. At 630 P.Mr. it suddenly kicked out, grew pale, and at 8 P M. it vomited. Next day (Saturday), at 3 P.M., it evacuated slimy blood, and this was the signal symptom that led to my seeing it. It was a well-developed boy of four months, but now lay in a state of extreme collapse on its mother's lap. It presented the following signs and symptoms, sufficiently distinct to make a diagnostic error impossible. Emesis frequent, the vomited matter resembling thin oil ; tenesmus, causing mucus, thin and pink, to ooze from the anus; anuria, from suppression, coincident with the earliest symptoms; flexion, with adduction of left thigh ; tympanites, central and superior; abdominal tumour, in position, deep-seated, oblique, and extending for about three inches from the left iliac region, upwards and to the right; rectal tumour, smooth and even, fixed and elastic, becoming tense during efforts at straining. This tumour was manifestly the tangible end of the abdominal swelling.

Treatment.-Abdominal taxis; large oil enemata; bella donna inunctions; nutriment suitable as to time, kind, and quantity. An operation was objected to. Death by syncope took place at 10 A.M. on Tuesday.

Necropsy forty-eight hours after death.-Distended small intestine, bulged up from incision; large intestine absent from its normal position; hidden by small intestine lay the tumour recognised during life, to all intents a livid sausage composed of the first two inches of the ileum, and the whole of the colon to within an inch and a half of the anus. The ileum had not passed the ilio-cæcal valve. The intussusception had been produced by the invagination of the upper into the lower part of the gut. Between the outer and middle walls of the mass was a quantity of blood-stained mucus; between the middle and inner were strangulated vessels and adherent peritoneum The mucous surfaces were swollen, soft, and moist; the muscular tissue softened and fragile, the serous surfaces when drawn asunder were abraded. The exciting canse of this case seems to have been the administration of the commonest and safest of all laxatives, a drug which acts on intestinal gland and muscular fibre. The analogue of the case is seen in true prolapsus ani produced by straining its factors were these. An empty canal, a relaxed mucosa, and abnormal activity of intestinal muscular fibre. The gaseous contents of the bowel probably play an important part in preventing invagination and other forms of intestinal obstruction. The sphineters of the rectum favour prolapse by constricting the part of the falling bowel which they encircle, while the longitudinal fibres labour in its expulsion. The rectal sphincters are foreshadowed far up the colon by the accumulation of circular muscular fibres between its saccules. I have seen a case of invagination of the colon reduced by an extensive rectal prolapse, which latter was afterwards reduced and a cure effected. Stoke Newington, $\mathbf{N}$. 